Sharif University of Technology
Scientia Iranica
Transactions E: Industrial Engineering
SCIENTIA

\title{
Multidimensional knapsack problem based on uncertain measure
}

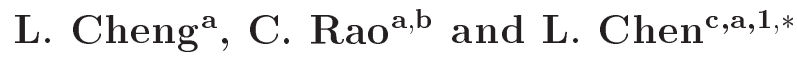 \\ a. College of Mathematics and Physics, Huanggang Normal University, Hubei 438000, China. \\ b. School of Science, Wuhan University of Technology, Wuhan 430070, China. \\ c. College of Mathematics and Sciences, Shanghai Normal University, Shanghai 200234, China.
}

Received 29 August 2015; received in revised form 6 June 2016; accepted 5 September 2016

\section{KEYWORDS}

Multidimensional knapsack problem; Uncertain network optimization;

Uncertain measure;

Discount constraint.

\begin{abstract}
The researchers of classical multidimensional knapsack problem have always assumed that the weights, values, and capacities are constant values. However, in the real-life industrial engineering applications, the multidimensional knapsack problem often comes with uncertainty about a lack of information about these parameters. This paper investigates a constrained multidimensional knapsack problem under uncertain environment, in which the relevant parameters are assumed to be uncertain variables. Within the framework of uncertainty theory, two types of uncertain programming models with discount constraints are constructed for the problem with different decision criteria, including the expected value criterion and the critical value criterion. Taking full advantage of the operational law for uncertain variables, the proposed models can be transformed into their corresponding deterministic models. After theoretically investigating the properties of the models, we do some numerical experiments. The numerical results illustrate that the proposed models are feasible and efficient for solving the constrained multidimensional knapsack problem with uncertain parameters.

(C) 2017 Sharif University of Technology. All rights reserved.
\end{abstract}

\section{Introduction}

The Multidimensional Knapsack Problem (MKP) is one of the most extensively-studied problems in network optimization. The task of MKP is to choose a subset of items in which the total value is maximized while the weight does not exceed the specified capacity of each dimension. MKP offers many practical applications in industrial engineering, such as production planning [1], project scheduling [2], service center location [3], and supply chain management [4], etc.

1. Present address: College of Management and Economics, Tianjin University, Tianjin 300072, China.

*. Corresponding author.

E-mail address: chenlinalbert@126.com (L. Chen)

doi: $10.24200 /$ sci. 2017.4485
For more comprehensive development of MKP, please consult Freville [5]. Due to its wide range of applicability, the multidimensional knapsack problem was widely studied in deterministic environment. However, in practice, some indeterminacy factors might appear in the problems due to the lack of history data, insufficient information, or some other reasons. As previous studies $[6,7]$ pointed out, the available capacity or weight might be unknown due to delay in previous jobs, or the item rewards might depend on market fluctuations. Thus, it is not suitable to use the classical models to investigate the multidimensional knapsack problem in these situations.

Several researchers deemed that such indeterminacy behaves like randomness. Based on this assumption, a lot of studies have been investigated within the framework of probability theory. For instance, Kosuch [8] studied a two-stage stochastic 
knapsack problem with random weights. Feng and Hartman [9] investigated a stochastic knapsack problem with homogeneous-sized items and postponement options. Han et al. [10] considered a certain class of chance-constrained knapsack problems where each item has an independent normally-distributed random weight. For more research on the stochastic knapsack problem, see Kleywegt and Papastavrou [7], Gibson et al. [11], Merzifonluoglu et al. [12], and others.

It is undeniable that probability theory is a useful tool to deal with indeterminacy factors. However, as is known to all of us, a fundamental premise of applying probability theory is that the estimated probability distribution is close enough to the true frequency. Thus, we have to rely on a great quantity of historical data and employ statistics to get the probability distribution. In the real-world, however, there exist many uncertain factors which cannot be ignored when the decision-makers make their decisions. Motivated by this consideration, several researchers have employed fuzzy set theory to deal with nondeterministic parameters for multidimensional knapsack problem. Okada and Gen [13] proposed the multiple-choice knapsack problems under fuzzy environment. Changdar et al. [14] considered an improved genetic algorithm to solve constrained knapsack problem with fuzzy parameters. Changdar et al. [15] investigated the knapsack problem with imprecise weight using genetic algorithms. Chen [16] proposed a parametric programming approach to analyze the fuzzy maximum total return in the continuous knapsack problem with fuzzy objective weights. Baykasoğlu and Ozsoydan [17] developed firefly algorithm to solve dynamic multidimensional knapsack problems. Kasperski and Kulej [18] considered the knapsack problem with imprecise profits and imprecise weights of items. Our paper differs from those of Okada and Gen [13] and Kasperski and Kulej [18] in the following aspects. First, we use an uncertain measure, which is a self-dual measure, to describe belief degree of the relevant parameter in the multidimensional knapsack problem. Based on this measure, the operational law in the uncertainty theory is simpler and easier than its counterpart in the fuzzy set theory. The details of operational law of uncertain variable are shown in Section 3. Second, we consider the multidimensional knapsack problem with a price-discount constraint. This type of multidimensional knapsack problem is commonly used in the fruit and/or vegetable retailing systems. Motivated by this discount strategy, our paper establishes a medium between the existing uncertainty theory and multidimensional knapsack problem.

However, if the uncertain factor comes from the decision-maker's empirical estimation, then it is not suitable to employ random variable or fuzzy variable to describe this kind of uncertain factor. In such cases, we have to invite some domain experts to give the belief degrees of the weights, values, and capacities. According to Kahneman and Tversky [19], human beings usually overweight unlikely events, thus the belief degree based on experts' estimations may be far from the cumulative frequency. One the one hand, in 2012, Liu [20] pointed out that if we insist on dealing with the belief degree by using probability theory in this situation, some counter-intuitive phenomena might occur. On the other hand, the fuzzy set theory is not self-consistent in mathematics and may lead to several counter-intuitive paradoxes in practice [21]. The details of counterintuitive paradoxes will be given in Section 2. Therefore, in this situation, we have no choice but to use the uncertainty theory, founded by Liu [22], to deal with the belief degree in the multidimensional knapsack problems.

Although multidimensional knapsack problems have been widely investigated, it has not been studied within the framework of uncertainty theory. This is because the uncertainty theory is a quite recent and new tool to deal with the belief degree mathematically. To the best of our knowledge, only Peng and Zhang [23] analyzed the uncertain knapsack problem with singlecapacity constraint. Following the main literature in the field of uncertainty theory, in their paper [23], the values and the weights were characterized as uncertain variables. But, in the real-life decision-making problems, the decision-makers usually encounter the situations of the knapsack problem with multiplecapacity constraints. Since no one has investigated the multidimensional knapsack problem under uncertain environment before, we have made attempts to fill this gap. Specifically, this paper considers a constrained multidimensional knapsack problem, which is usually encountered in the fruit and/or vegetable retailing systems based on uncertain measure. We employed two kinds of uncertain variables, i.e. linear uncertain and zigzag uncertain variables, to characterize the weights, values, and capacities of the multidimensional knapsack problem with a discount constraint. Within the framework of uncertainty theory, two types of uncertain programming models are constructed for the problem with different criteria, e.g. the expected value and the critical value criteria. Based on the operational law for uncertain variables, some theoretical analyses are provided. The theoretical results illustrate that the maximum value of chance-constrained programming model actually decrease with respect to the predetermined confidence level.

The structure of this paper is as follows. In Section 2, we state the fact that the fuzzy set theory cannot be employed to model the imprecise parameters in multidimensional knapsack problem. To facilitate the understanding of the paper, some basic concepts and results related to uncertainty theory are outlined and the classical multidimensional knapsack problem is 
briefly reviewed in Section 3. Section 4 proposes two types of uncertain programming models for uncertain multidimensional knapsack problem and transforms the models into their corresponding deterministic forms within the framework of uncertainty theory. Some numerical examples are given to show the applications of the proposed models in Section 5. Section 6 gives some concluding remarks and possible directions for the future work.

\section{Why using uncertain measure?}

In this section, we present the fact that the fuzzy set theory cannot be employed to model the imprecise weights, imprecise values, and imprecise capacities in multidimensional knapsack problem under uncertain environment.

As discussed in the introduction, the previous studies of using the fuzzy set theory to investigate the multidimensional knapsack problem have indeed opened a new research perspective for solving multidimensional knapsack problems with imprecise parameters given by experts' estimations. However, with a deeper research on the problem, we find out that several counterintuitive paradoxes will appear if fuzzy variables are employed to characterize the imprecise parameters of MKP.

We take the imprecise capacities of MKP as an example to illustrate several counterintuitive paradoxes when we use the fuzzy set theory to study the multidimensional knapsack problem. Supposing that the capacity of the different knapsack is regarded as a fuzzy number, we have a membership function to describe it. In the fuzzy set theory, possibility measure (Pos) and necessity measure (Nec) are the two basic measures. Assume that the membership function of knapsack capacity is a triangular fuzzy variable, $\xi \sim(8,9,10)$. Then, we can obtain the following two paradoxes. First, based on the membership function, it is known from possibility theory that $\operatorname{Pos}\{\xi=9\}=\operatorname{Pos}\{\xi \neq 9\}$ and $\operatorname{Nec}\{\xi=9\}=\operatorname{Nec}\{\xi \neq 9\}$. In other words, the capacity of knapsack, being exactly 9 and not exactly 9 , has the same belief degree in possibility measure and necessity measure. This implies that the two events will happen equally likely. This conclusion is obviously unreasonable. Second, the capacity of knapsack is exactly 9 with belief degree 1 in possibility measure. However, this conclusion is also hard to accept because the belief degree of exactly 9 should be almost zero.

It can be seen from the above two counterintuitive examples that the fuzzy set theory is not a suitable tool to model the imprecise weights, imprecise values, and imprecise capacities in multidimensional knapsack problem when the relevant parameters are given by experts' estimations. In order to handle this difficulty, Liu [22] introduced an uncertain measure within the framework of uncertainty theory. When we use the uncertain measure to gauge the subjective uncertain quantities, the abovementioned paradoxes will disappear immediately. In fact, the uncertainty theory has been widely applied to various real-life industrial engineering problems, e.g. knapsack problem with single capacity [23], optimal assignment problem [24], the shortest path problem [25], railway transportation planning problem [26], supply chain contract design problem [27], and so on. In the next section, we will introduce the uncertainty theory briefly.

\section{Preliminary}

In this section, some basic concepts and results of uncertainty theory are recalled, and the classical multidimensional knapsack problem is briefly reviewed.

\subsection{Uncertainty theory}

Uncertainty theory was founded by Liu [22] in 2007 and refined by Liu [21] in 2010. The uncertain programming, established by Liu [28], was a type of mathematical programming involving uncertain variables. In recent years, several scholars have employed uncertain programming to investigate the real-life decisionmaking problems. For instance, Gao [25] studied the uncertainty distribution of the shortest path length and proposed an effective method to find the $\alpha$-shortest path and the shortest path in an uncertain network. Zhang and Peng [24] employed uncertain programming to deal with uncertain optimal assignment problem in which profit is an uncertain variable. Recently, Peng et al. [29] presented a state-of-the-art review on the recent advances in the area of uncertain network optimization.

Assume that $\Gamma$ is a nonempty set, and $\mathcal{L}$ is a $\sigma$ algebra over $\Gamma$. Each element $\Lambda$ in $\mathcal{L}$ is called an event.

A set function $\mathcal{M}: \mathcal{L} \rightarrow[0,1]$ is called an uncertain measure if it satisfies the following three axioms [22]:

Axiom 1 (normality axiom). $\mathcal{M}\{\Gamma\}=1$ for the universal set $\Gamma$;

Axiom 2 (duality axiom). $\mathcal{M}\{\Lambda\}+\mathcal{M}\left\{\Lambda^{c}\right\}=1$ for any event $\Lambda \in \mathcal{L}$;

Axiom 3 (subadditivity axiom). For every countable sequence of events $\Lambda_{1}, \Lambda_{2}, \cdots$, we have:

$$
\mathcal{M}\left\{\bigcup_{i=1}^{\infty} \Lambda_{i}\right\} \leq \sum_{i=1}^{\infty} \mathcal{M}\left\{\Lambda_{i}\right\} .
$$

Definition 1 [22]. Let $\Gamma$ be a nonempty set, $\mathcal{L}$ a $\sigma$ algebra over $\Gamma$, and $\mathcal{M}$ an uncertain measure. Then, the triplet $(\Gamma, \mathcal{L}, \mathcal{M})$ is called an uncertainty space.

Product uncertain measure $\mathcal{M}$ on product $\sigma$ algebra $\mathcal{L}$ was defined by Liu [30], thus producing the following product axiom. 
Axiom 4 (product axiom). Let $\left(\Gamma_{k}, \mathcal{L}_{k}, \mathcal{M}_{k}\right)$ be uncertainty spaces for $k=1,2, \cdots$ The product uncertain measure, $\mathcal{M}$, is an uncertain measure satisfying:

$$
\mathcal{M}\left\{\prod_{k=1}^{\infty} \Lambda_{k}\right\}=\bigwedge_{k=1}^{\infty} \mathcal{M}_{k}\left\{\Lambda_{k}\right\}
$$

where $\Lambda_{k}$ refers to arbitrarily chosen events from $\mathcal{L}_{k}$ for $k=1,2, \cdots$, respectively.

Uncertain variable is mainly used to model the uncertain quantities. A formal definition was given by Liu [22] as follows:

Definition 2 [22]. An uncertain variable is a function $\xi$ from an uncertainty space $(\Gamma, \mathcal{L}, \mathcal{M})$ to the set of real numbers such that $\{\xi \in B\}$ is an event for any Borel set $B$.

On the basis of uncertain measure, Liu [22] gave the definition of uncertainty distribution of an uncertain variable, $\xi$, which was defined by $\Phi(x)=\mathcal{M}\{\xi \leq$ $x\}$. If an uncertainty distribution, $\Phi(x)$, is a continuous and strictly increasing function with respect to $x$ at which $0<\Phi(x)<1$ and the following limitations hold:

$$
\lim _{x \rightarrow-\infty} \Phi(x)=0, \quad \lim _{x \rightarrow+\infty} \Phi(x)=1
$$

then, uncertainty distribution, $\Phi(x)$, is said to be regular [21].

Definition 3 [30]. Uncertain variables $\xi_{1}, \xi_{2}, \cdots, \xi_{n}$ are said to be independent if:

$$
\mathcal{M}\left\{\bigcap_{i=1}^{n}\left(\xi_{i} \in B_{i}\right)\right\}=\bigwedge_{i=1}^{n} \mathcal{M}\left\{\xi_{i} \in B_{i}\right\}
$$

for any Borel sets $B_{1}, B_{2}, \cdots, B_{n}$.

Theorem 1 [21]. Let $\xi_{1}, \xi_{2}, \cdots, \xi_{n}$ be independent uncertain variables with regular uncertainty distributions $\Phi_{1}, \Phi_{2}, \cdots, \Phi_{n}$, respectively. If function $f\left(x_{1}\right.$, $\left.\cdots, x_{m}, x_{m+1}, \cdots, x_{n}\right)$ is strictly increasing with respect to $x_{1}, x_{2}, \cdots, x_{m}$ and strictly decreasing with respect to $x_{m+1}, x_{m+2}, \cdots, x_{n}$, then the uncertain variable:

$$
\xi=f\left(\xi_{1}, \cdots, \xi_{m}, \xi_{m+1}, \cdots, \xi_{n}\right),
$$

has an inverse uncertainty distribution:

$$
\begin{gathered}
\Psi^{-1}(\alpha)=f\left(\Phi_{1}^{-1}(\alpha), \cdots, \Phi_{m}^{-1}(\alpha), \Phi_{m+1}^{-1}(1-\alpha),\right. \\
\left.\cdots, \Phi_{n}^{-1}(1-\alpha)\right) .
\end{gathered}
$$

Theorem 2 [21]. Let $\xi_{1}, \xi_{2}, \cdots, \xi_{n}$ be independent uncertain variables with regular uncertainty distributions $\Phi_{1}, \Phi_{2}, \cdots, \Phi_{n}$, respectively. If function $f\left(x_{1}\right.$, $\left.\cdots, x_{m}, x_{m+1}, \cdots, x_{n}\right)$ is strictly increasing with respect to $x_{1}, x_{2}, \cdots, x_{m}$ and strictly decreasing with respect to $x_{m+1}, x_{m+2}, \cdots, x_{n}$, then:

$$
\mathcal{M}\left\{f\left(\xi_{1}, \cdots, \xi_{m}, \xi_{m+1}, \cdots, \xi_{n}\right) \leq 0\right\} \geq \alpha
$$

if and only if:

$$
\begin{aligned}
f\left(\Phi_{1}^{-1}(\alpha), \cdots, \Phi_{m}^{-1}(\alpha), \Phi_{m+1}^{-1}(1-\alpha), \cdots,\right. \\
\left.\Phi_{n}^{-1}(1-\alpha)\right) \geq 0 .
\end{aligned}
$$

According to Theorems 1 and 2, we can easily obtain the inverse uncertainty distribution of function $f\left(\xi_{1}, \xi_{2}, \cdots, \xi_{n}\right)$. Therefore, we can transform an indeterminacy model into a deterministic form based on Theorems 1 and 2. On the basis of uncertain measure, Liu [22] defined the expected value of uncertain variables as follows. If $\xi$ be an uncertain variable, then the expected value of $\xi$ is defined by:

$$
E[\xi]=\int_{0}^{+\infty} \mathcal{M}\{\xi \geq r\} \mathrm{d} r-\int_{-\infty}^{0} \mathcal{M}\{\xi \geq r\} \mathrm{d} r,
$$

provided that at least one of the integrals was finite. If $\xi$ had an uncertainty distribution, $\Phi(x)$, Liu [22] verified that the expected value could be calculated by:

$$
E[\xi]=\int_{0}^{+\infty}(1-\Phi(x)) \mathrm{d} x-\int_{-\infty}^{0} \Phi(x) \mathrm{d} x .
$$

In addition, Liu [21] verified that if the uncertainty distribution, $\Phi(x)$, was regular, then $E[\xi]=\int_{0}^{1} \Phi^{-1}(\alpha) \mathrm{d} \alpha$.

Theorem 3 [21]. Assume that $\xi_{1}$ and $\xi_{2}$ are independent uncertain variables with the finite expected values. For any real numbers, $a_{1}$ and $a_{2}$, we have:

$$
E\left[a_{1} \xi_{1}+a_{2} \xi_{2}\right]=a_{1} E\left[\xi_{1}\right]+a_{2} E\left[\xi_{2}\right] .
$$

Example 1. Uncertain variable, $\xi$, is called zigzag if it has a zigzag uncertainty distribution (see Figure 1):

$$
\Phi(x)= \begin{cases}0, & \text { if } x \leq a \\ (x-a) / 2(b-a), & \text { if } a \leq x \leq b \\ (x+c-2 b) / 2(c-b), & \text { if } b \leq x \leq c \\ 1, & \text { if } x \geq c,\end{cases}
$$

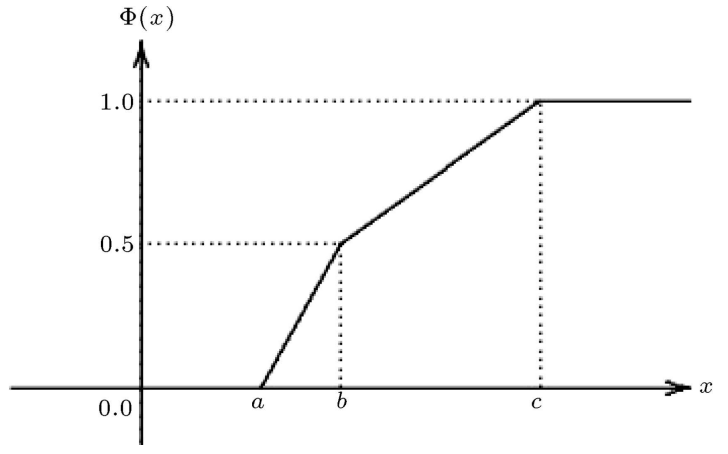

Figure 1. Zigzag uncertainty distribution. 


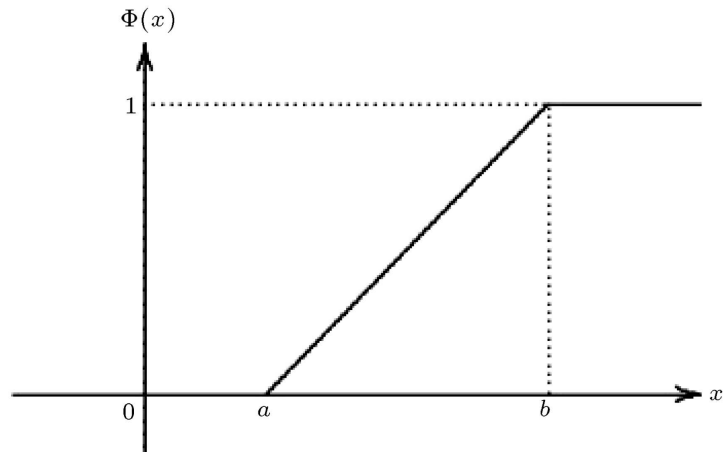

Figure 2. Linear uncertainty distribution.

denoted by $\mathcal{Z}(a, b, c)$, where $a, b$, and $c$ are real numbers with $a<b<c$. The inverse uncertainty distribution, $\Phi^{-1}(\alpha)$, of uncertain variable, $\xi$, is given by:

$$
\Phi^{-1}(\alpha)= \begin{cases}(1-2 \alpha) a+2 \alpha b, & \text { if } \alpha<0.5 \\ (2-2 \alpha) b+(2 \alpha-1) c, & \text { if } \alpha \geq 0.5\end{cases}
$$

The expected value of $\xi$ is $E[\xi]=(a+2 b+c) / 4$.

Example 2. Assume that $\xi \sim \mathcal{L}(a, b)$ is a linear uncertain variable, where $a$ and $b$ are real numbers with $a<b$. Its uncertainty distribution, $\Phi(x)$, (see Figure 2) and inverse uncertainty distribution, $\Phi^{-1}(\alpha)$, are given by:

$$
\Phi(x)= \begin{cases}0, & \text { if } x \leq a \\ (x-a) /(b-a), & \text { if } a \leq x \leq b \\ 1, & \text { if } x \geq b\end{cases}
$$

and:

$$
\Phi^{-1}(\alpha)=(1-\alpha) a+\alpha b
$$

respectively. The expected value of $\xi$ is $E[\xi]=(a+b) / 2$.

\subsection{Classical multidimensional knapsack problem with discount constraint}

In this section, we present the formulation of Multidimensional Knapsack Problem (MKP) with discount constraint in deterministic environment. Generally speaking, MKP is concerned with a set of items, $N=\{1,2, \cdots, n\}$, to be packed in a knapsack that has a set of dimensions, $M=\{1,2, \cdots, m\}$. The maximum capacity of each dimension, $i$, is denoted as $c_{i}, i=1,2, \cdots, m$. Each item, $j \in N$, has a weight, $w_{i j}$, in each dimension, and a corresponding value $v_{j}$, $i=1,2, \cdots, m, j=1,2, \cdots, n$. The task of MKP is to choose a subset of items that the total value is maximized, while the weight does not exceed specified capacity, $c_{i}$, of each dimension, $i$. Motivated by various examples in the real-life decision making problems, we consider the MKP with discount constraint. For example, some of the objects are available to which discount has been offered if the selected quantity is greater than a predetermined level. The fruit and/or vegetable retailing systems exhibit the characteristic where the wholesalers adopt the discount strategy. If the minimum predetermined amount of object is selected, then a discount is to be considered for that item and the total discount must reach or exceed a predetermined total discount, $D$. For $j$ th item, if minimum, $m_{j}$, unit of object is consumed, the discount is $d_{j}$; otherwise, no discount is considered and the total predetermined minimum discount is $D$. Therefore, we construct the mathematical model for multidimensional knapsack problem with discount constraint as follows:

$$
\max \sum_{j=1}^{n} v_{j} x_{j}
$$

subject to:

$$
\begin{array}{ll}
\sum_{j=1}^{n} w_{i j} x_{j} \leq c_{i}, & \text { for } i=1,2, \cdots, m, \\
\sum_{j=1}^{n} d_{j} y_{j} \geq D, & \\
x_{j} \in\{0,1\}, y_{j} \in\{0,1\}, \quad \text { for } j=1,2, \cdots, n .
\end{array}
$$

It is necessary to point out that the first constraint requires that the knapsacks not exceed the capacity of any knapsack. The second constraint is the discount constraint. Variables $x_{j}$ and $y_{j}$ are all binary variables. The detailed characteristics of these two binary variables are as follows. If the $j$ th item is selected, then $x_{j}=1$; otherwise $x_{j}=0$. If $x_{j} \geq m_{j}\left(m_{j}\right.$ take value from $[0,1])$ and $d_{j}>0$, then $y_{j}=1$; otherwise $y_{j}=0$.

\section{Uncertain multidimensional knapsack models}

Model (1) assumes that the weights, values, and capacities are constant values. However, in practice, these parameters are not usually constant values. As discussed in the introduction, we always lack history data, or even history data are invalid. Then, one problem is naturally produced; that is, how can we deal with this kind of indeterminacy factor in the multidimensional knapsack problem? In this situation, the relevant data can only be obtained from the decision-makers' empirical estimations in a practical way. As mentioned before, the uncertainty theory provides a new tool to deal with uncertain information, especially subjective or empirical information. Hence, in this paper, we assume that each item, $j$, has an uncertain weight, $\eta_{i j}$, in each dimension and an uncertain value, $\xi_{j}$; each 
capacity of any knapsack is an uncertain variable, $\zeta_{i}$, and the discount of the $j$ th item is also an uncertain variable $\tau_{j}, i=1,2, \cdots, m, j=1,2, \cdots, n$.

Without loss of generality, we also assume that the parameters are nonnegative and independent uncertain variables. Such an independent assumption is extensively used in a recent work in the area of uncertain network optimization (Gao [25], Zhang and Peng [24], and Peng et al. [29], to name a few). For the sake of convenience, all the uncertain values, uncertain weights, uncertain capacities, and uncertain discount coefficients are presented by uncertain vectors $\boldsymbol{\xi}=\left(\xi_{1}, \xi_{2}, \cdots, \xi_{n}\right), \boldsymbol{\eta}=\left(\eta_{i j} \mid i=\right.$ $1,2, \cdots, m, j=1,2, \cdots, n), \boldsymbol{\zeta}=\left(\zeta_{1}, \zeta_{2}, \cdots, \zeta_{m}\right)$ and $\boldsymbol{\tau}=\left(\tau_{1}, \tau_{2}, \cdots, \tau_{n}\right)$, respectively. Then, the uncertain multidimensional knapsack can be denoted by $K=$ $(V, \boldsymbol{\xi}, \boldsymbol{\eta}, \boldsymbol{\zeta}, \boldsymbol{\tau})$ in which $V$ is the maximum value by which the knapsack can be carried out. Then, the total value of maximum pack scheme of uncertain multidimensional knapsack is $f(\xi)=\sum_{j=1}^{n} \xi_{j} x_{j}$.

It is clear that $f(\xi)$ is also an uncertain variable because uncertain values $\xi_{j}$ are all uncertain variables for $j=1,2, \cdots, n$. Then, the Uncertain Multidimensional Knapsack Problem (UMKP) can be expressed as follows:

$$
\max \sum_{j=1}^{n} \xi_{j} x_{j}
$$

subject to:

$$
\begin{aligned}
& \sum_{j=1}^{n} \eta_{i j} x_{j}-\zeta_{i} \leq 0, \quad \text { for } i=1,2, \cdots, m, \\
& \sum_{j=1}^{n} \tau_{j} y_{j} \geq D, \\
& x_{j} \in\{0,1\}, y_{j} \in\{0,1\}, \quad \text { for } j=1,2, \cdots, n .
\end{aligned}
$$

Model (2) turns meaningless in the sense of mathematical viewpoints under uncertain environment. In order to optimize the objective, it is inevitable for us to rank uncertain variables according to some decision criteria. Generally speaking, there exist two kinds of decision criteria to rank the uncertain variables according to the manager's philosophy of modeling uncertainty. The two kinds of decision criteria are the expected value criterion and the critical value criterion which are based on the manager's different risk attitude. On the one hand, the main idea of the expected value criterion is to optimize the expected value of the manager's objective. On the other hand, the essential idea of the critical value criterion is to optimize the critical value of the manager's objective with predetermined confidence level. In the next section, we will employ these two kinds of criteria to rank the uncertain variables and construct two kinds of uncertain programming models with discount constraint. One is the expected value model, and another is the chance-constrained programming model.

\subsection{Expected value model}

Different from a deterministic environment, the total value for UMKP is an uncertain variable under uncertain environment. It is difficult for us to rank two uncertain variables directly. The first way is to adopt the expected value criterion to rank the uncertain variable. Therefore, the first type of uncertain programming, i.e. the so-called expected value model, is constructed based on this kind of ranking criterion. The main idea of the expected value model is to optimize the expected value of objective function subject to some expected constraints. Motivated by this most understandable method for modeling the real-life industrial engineering problems, we present the expected value model for UMKP as follows:

$$
\max E\left[\sum_{j=1}^{n} \xi_{j} x_{j}\right],
$$

subject to:

$$
\begin{aligned}
& E\left[\sum_{j=1}^{n} \eta_{i j} x_{j}-\zeta_{i} \leq 0\right], \quad \text { for } i=1,2, \cdots, m, \\
& E\left[\sum_{j=1}^{n} \tau_{j} y_{j}\right] \geq D, \\
& x_{j} \in\{0,1\}, y_{j} \in\{0,1\}, \quad \text { for } j=1,2, \cdots, n .
\end{aligned}
$$

We can see that Model (3) is constructed under the light of uncertain environment. To seek the optimal solution of Model (3), it is necessary for us to compute expected values of the relevant parameters. In order to solve the model easily, it is better for us to convert the model into its crisp equivalent model. Taking full advantage of the concept of expected value for uncertain variable mentioned in Section 3, we can easily obtain the corresponding equivalent model of Model (3). In the following, we shall discuss this issue.

Theorem 4. If $\xi_{j}, \eta_{i j}, \zeta_{i}$, and $\tau_{j}$ are independent uncertain variables with regular uncertainty distributions $\Phi_{j}, \Psi_{i j}, \Upsilon_{i}$, and $F_{j}, i=1,2, \cdots, m, j=1,2, \cdots, n$, respectively. Then, Model (3) is equivalent to the following model:

$$
\max \sum_{j=1}^{n} x_{j} \int_{0}^{1} \Phi_{j}^{-1}(\alpha) \mathrm{d} \alpha
$$


subject to:

$$
\begin{aligned}
& \sum_{j=1}^{n} x_{j} \int_{0}^{1} \Psi_{i j}^{-1}(\alpha) \mathrm{d} \alpha \leq \int_{0}^{1} \Upsilon_{i}^{-1}(\alpha) \mathrm{d} \alpha, \\
& \quad \text { for } i=1,2, \cdots, m, \\
& \sum_{j=1}^{n} y_{j} \int_{0}^{1} F_{j}^{-1}(\alpha) \mathrm{d} \alpha \geq D, \\
& x_{j} \in\{0,1\}, y_{j} \in\{0,1\}, \quad \text { for } j=1,2, \cdots, n,
\end{aligned}
$$

where $\Phi_{j}^{-1}, \Psi_{i j}^{-1}, \Upsilon_{i}^{-1}$, and $F_{j}^{-1}$ are the inverse uncertainty distributions of $\xi_{j}, \eta_{i j}, \zeta_{i}$, and $\tau_{j}$, for $i=1,2, \cdots, m, j=1,2, \cdots, n$, respectively.

Proof. It follows from Theorem 3 that the expected value of pack scheme can be rewritten as:

$$
E\left[\sum_{j=1}^{n} \xi_{j} x_{j}\right]=\sum_{j=1}^{n} x_{j} \int_{0}^{1} \Phi_{j}^{-1}(\alpha) \mathrm{d} \alpha .
$$

Similarly, the remaining uncertain constraints can be transformed into their corresponding deterministic constraints. Therefore, the proof of the theorem is complete.

\subsection{Chance-constrained programming model}

The expected value model depends only on the expected values of uncertain variables, but variance is not considered in the process of the decision making. This may lead to the risk of making a bad decision. In order to avoid such a risk, many decision-makers may first set a confidence level, $\alpha \in(0,1)$, as a safety margin. What's more, in many situations, the managers want to make decisions in the case of the knapsack value wants satisfying some chance constraints with at least some given confidence level which reflects his/her attitude for the risk during the decision-making process.

As we know, the essential idea of the critical value criterion is to maximize the critical value of the relevant parameters with predetermined confidence levels. Therefore, based on the critical value criterion, the chance-constrained programming was pioneered by Charnes and Cooper [31]. Chance-constrained programming deals with uncertainty by specifying the desired levels of confidence with the chance constraints and offers another effective method of modeling uncertain decision systems. It offers a powerful means of modeling uncertain decision systems with the assumption that the uncertain constraints will hold at least confidence level, $\alpha$.

As for UMKP in this paper, if the decisionmaker prefers to treat the problem under the chance constraints, then the model can be constructed as follows:

$\max V$,

subject to:

$$
\begin{aligned}
& \mathcal{M}\left\{\sum_{j=1}^{n} \xi_{j} x_{j} \geq V\right\} \geq \alpha \\
& \mathcal{M}\left\{\sum_{j=1}^{n} \eta_{i j} x_{j} \leq \zeta_{i}\right\} \geq \beta, \quad \text { for } i=1,2, \cdots, m \\
& \mathcal{M}\left\{\sum_{j=1}^{n} \tau_{j} y_{j} \geq D\right\} \geq \gamma, \\
& x_{j} \in\{0,1\}, y_{j} \in\{0,1\}, \quad \text { for } j=1,2, \cdots, n,
\end{aligned}
$$

where $\alpha, \beta$, and $\gamma$ are the predetermined confidence levels.

Theorem 5. If $\xi_{j}, \eta_{i j}, \zeta_{i}$, and $\tau_{j}$ are independent uncertain variables with regular uncertainty distributions $\Phi_{j}, \Psi_{i j}, \Upsilon_{i}$, and $F_{j}, i=1,2, \cdots, m, j=1,2, \cdots, n$, respectively. Then, Model (5) is equivalent to the following model:

$\max V$,

subject to:

$$
\begin{aligned}
& \sum_{j=1}^{n} x_{j} \Phi_{j}^{-1}(1-\alpha) \geq V, \\
& \sum_{j=1}^{n} x_{j} \Psi_{i j}^{-1}(\beta) \leq \Upsilon_{i}^{-1}(1-\beta), \quad \text { for } i=1,2, \cdots, m, \\
& \sum_{j=1}^{n} y_{j} F_{j}^{-1}(1-\gamma) \geq D, \\
& x_{j} \in\{0,1\}, y_{j} \in\{0,1\}, \quad \text { for } j=1,2, \cdots, n,
\end{aligned}
$$

where $\Phi_{j}^{-1}, \Psi_{i j}^{-1}, \Upsilon_{i}^{-1}$, and $F_{j}^{-1}$ are the inverse uncertainty distributions of $\xi_{j}, \eta_{i j}, \zeta_{i}$, and $\tau_{j}$, respectively.

Proof. By using the inverse uncertainty distribution, constraint $\mathcal{M}\left\{\sum_{j=1}^{n} \xi_{j} x_{j} \geq V\right\} \geq \alpha$ converted into a deterministic constraint $\sum_{j=1}^{n} x_{j} \Phi_{j}^{-1}(1-\alpha) \geq V$. Similarly, the remaining uncertain constraints can be transformed into their corresponding deterministic constraints. Therefore, the proof of the theorem is complete. 
Since the objective of Model (6) is a function of the confidence level, $\alpha$, the relationship between the optimal objective value and parameter $\alpha$ should be investigated. The following theorem will answer this research question.

Theorem 6. The optimal objective value of model (6) is non-increasing with respect to confidence level, $\alpha$.

Proof. Suppose that the feasible domain of Model (6) is denoted by $I$, and the corresponding optimal objective value is denoted by $V$. Without loss of generality, we assume $\alpha_{1} \geq \alpha_{2}$. Let $V_{1}$ and $V_{2}$ be the corresponding optimal objective values of Model (6). Then, we have:

$$
V_{1}=\sum_{v_{i} \in V} x_{i} \Phi_{i}^{-1}\left(1-\alpha_{1}\right) \leq \sum_{v_{i} \in V} x_{i} \Phi_{i}^{-1}\left(1-\alpha_{2}\right)=V_{2} .
$$

Thus, the proof is completed.

It is worth noting that Models (4) and (6) are both 0-1 integer programming models. Generally speaking, solving such models requires much computational effort. As mentioned earlier, however, with the aid of some well-developed optimization solvers, such as LINGO, BARON, and CPLEX, we can solve Models (4) and (6) easily and effectively. Like the previous studies [23-25], we solve Models (4) and (6) by using LINGO 5 software.

\section{Numerical example}

In order to show the application of the models, two numerical examples are given in this section. To solve the above models, the developed optimization software solver LINGO 11.0 will be employed in the examples to produce the optimal solutions. The computational experiments are performed on a personal computer (Dell with Intel(R) Core(TM) i5-2450 M CPU 2.50 GHZ and RAM 2.50 GB).

Example 3. Suppose that there are eight items and four knapsacks. Each item has a weight and a value in each dimension, and the weight of each knapsack is different. The task is to find a pack scheme that yields the maximum total value under the capacity constraints of the different knapsacks. At the beginning of this task, the decision-maker needs to obtain the basic data, such as the weights, values, and capacities of the MKP. However, due to economic reasons or technical difficulties, the decision-maker cannot always get these data exactly. For this condition, the usual way is to obtain the uncertain data by means of experience evaluation or expert advice. It should be noted that there are several researchers who characterize the relevant parameters as fuzzy variables, such as Okada and Gen [13], Kasperski and Kulej [18], and Kasperski and Zieliński [32]. However, as pointed out in Section 2, the fuzzy set theory is not a suitable tool to model the imprecise weights, imprecise values, and imprecise capacities in the multidimensional knapsack problem when the relevant parameters are given by domain experts. Therefore, in this paper, we assume that the uncertain parameters are all independent uncertain variables.

It is necessary to point out that although the functional form of the uncertain variables are a little similar to the fuzzy variables, the theoretical foundations are different. One the one hand, the distribution functional graphs of zigzag uncertain variable and linear uncertain variable are shown in Figures 1 and 2, respectively. One can find that the distribution functional forms of uncertain variables are quite different from those of fuzzy variables. On the other hand, the two theoretical foundations are the uncertainty theory and the fuzzy set theory, respectively. We study the uncertain multidimensional knapsack problem within the framework of uncertain programming, while the paper [18] investigates the fuzzy knapsack problem based on the fuzzy programming, so our numerical example is quite different from the example given in [18].

Like the previous studies (Zhang and Peng [24], Gao [25], and Peng et al. [29], to name a few), we assume that values $\xi_{j}$ of eight items and capacities $\zeta_{i}$ of four knapsacks are independent zigzag uncertain variables, which are listed as follows:

$$
\begin{aligned}
\xi_{1} & \sim \mathcal{Z}(24,26,28), & & \xi_{2} \sim \mathcal{Z}(23,24,25), \\
\xi_{3} & \sim \mathcal{Z}(20,22,24), & & \xi_{4} \sim \mathcal{Z}(23,25,27), \\
\xi_{5} & \sim \mathcal{Z}(29,31,33), & \xi_{6} & \sim \mathcal{Z}(27,28,29), \\
\xi_{7} & \sim \mathcal{Z}(23,26,29), & \xi_{8} & \sim \mathcal{Z}(30,32,34), \\
\zeta_{1} & \sim \mathcal{Z}(32,62,92), & \zeta_{2} & \sim \mathcal{Z}(37,77,97), \\
\zeta_{3} & \sim \mathcal{Z}(50,80,110), & \zeta_{4} & \sim \mathcal{Z}(60,80,100) .
\end{aligned}
$$

Additionally, all weights, $\eta_{i j}$, of each item in each dimension are listed in Table 1 . Let items $I_{1}, I_{3}, I_{5}$, and $I_{7}$ be considered for discount with the minimum weights as $70 \%, 80 \%, 85 \%$, and $90 \%$ of the total available objects, respectively. So, mathematically, $m_{1}=0.7, m_{3}=0.8, m_{5}=0.85$, and $m_{7}=0.9$. The total discount is $D=6$. We assume that similar to the item values and knapsack capacities, the discounts are also zigzag uncertain variables, which are listed as 
Table 1. The weights $\eta_{i j}$ of each item in each dimension.

\begin{tabular}{ccccc}
\hline Items/dimensions & $\mathbf{1}$ & $\mathbf{2}$ & $\mathbf{3}$ & $\mathbf{4}$ \\
\hline 1 & $\mathcal{Z}(5,13,19)$ & $\mathcal{Z}(6,8,11)$ & $\mathcal{Z}(7,12,15)$ & $\mathcal{Z}(5,9,13)$ \\
2 & $\mathcal{Z}(5,9,13)$ & $\mathcal{Z}(10,12,13)$ & $\mathcal{Z}(5,8,12)$ & $\mathcal{Z}(10,12,15)$ \\
3 & $\mathcal{Z}(8,12,14)$ & $\mathcal{Z}(8,13,17)$ & $\mathcal{Z}(7,12,13)$ & $\mathcal{Z}(6,9,13)$ \\
4 & $\mathcal{Z}(5,9,13)$ & $\mathcal{Z}(12,14,16)$ & $\mathcal{Z}(10,13,17)$ & $\mathcal{Z}(7,12,15)$ \\
5 & $\mathcal{Z}(6,9,13)$ & $\mathcal{Z}(7,12,15)$ & $\mathcal{Z}(10,13,15)$ & $\mathcal{Z}(5,7,10)$ \\
6 & $\mathcal{Z}(10,12,17)$ & $\mathcal{Z}(8,10,15)$ & $\mathcal{Z}(10,11,14)$ & $\mathcal{Z}(17,18,23)$ \\
7 & $\mathcal{Z}(10,13,18)$ & $\mathcal{Z}(5,10,13)$ & $\mathcal{Z}(11,14,19)$ & $\mathcal{Z}(11,15,19)$ \\
8 & $\mathcal{Z}(10,12,16)$ & $\mathcal{Z}(5,10,14)$ & $\mathcal{Z}(17,18,19)$ & $\mathcal{Z}(17,20,22)$ \\
\hline
\end{tabular}

follows:

$$
\begin{aligned}
\tau_{1} & \sim \mathcal{Z}(3,4,5), & & \tau_{2} \sim \mathcal{Z}(4,6,8), \\
\tau_{3} & \sim \mathcal{Z}(5,6,7), & & \tau_{4} \sim \mathcal{Z}(8,9,10), \\
\tau_{5} & \sim \mathcal{Z}(7,8,9), & & \tau_{6} \sim \mathcal{Z}(5,6,7), \\
\tau_{7} & \sim \mathcal{Z}(4,7,10), & & \tau_{8} \sim \mathcal{Z}(6,8,10) .
\end{aligned}
$$

According to Theorem 4, the expected value model for UMKP is equivalent to the following one:

$$
\max \sum_{j=1}^{8} x_{j} \int_{0}^{1} \Phi_{j}^{-1}(\alpha) \mathrm{d} \alpha
$$

subject to:

$$
\begin{aligned}
& \sum_{j=1}^{8} x_{j} \int_{0}^{1} \Psi_{i j}^{-1}(\alpha) \mathrm{d} \alpha \leq \int_{0}^{1} \Upsilon_{i}^{-1}(\alpha) \mathrm{d} \alpha, \\
& \quad \text { for } i=1,2,3,4, \\
& \sum_{j=1}^{8} y_{j} \int_{0}^{1} F_{j}^{-1}(\alpha) \mathrm{d} \alpha \geq D, \\
& x_{j} \in\{0,1\}, y_{j} \in\{0,1\}, \quad \text { for } j=1,2, \cdots, 8 .
\end{aligned}
$$

Taking by LINGO, we obtain the optimal solution to Model (7) as follows:

$$
\left(x_{1}, x_{2}, x_{3}, x_{4}, x_{5}, x_{6}, x_{7}, x_{8}\right)^{T}=(0,0,0,1,1,1,0,1)^{T},
$$

i.e. the decision-maker should carry goods 4, 5, 6, and 8 into the bag, and the expected maximum total value of the items that can be carried in the bag is 116 .

Assume that the confidence levels are $\alpha=\beta=$ $\gamma=0.9$. According to Theorem 5, the chanceconstrained programming model for UMKP is equivalent to the following one:

$\max V$ subject to:

$$
\begin{aligned}
& \sum_{j=1}^{8} x_{j} \Phi_{j}^{-1}(0.1) \geq V \\
& \sum_{j=1}^{8} x_{j} \Psi_{i j}^{-1}(0.9) \leq \Upsilon_{i}^{-1}(0.1), \quad \text { for } i=1,2,3,4, \\
& \sum_{j=1}^{8} y_{j} F_{j}^{-1}(0.1) \geq D, \\
& x_{j} \in\{0,1\}, y_{j} \in\{0,1\}, \quad \text { for } j=1,2, \cdots, 8 .
\end{aligned}
$$

Using LINGO software, we obtain the optimal solution to Model (8) as follows:

$$
\left(x_{1}, x_{2}, x_{3}, x_{4}, x_{5}, x_{6}, x_{7}, x_{8}\right)^{T}=(0,0,0,1,1,1,0,1)^{T},
$$

i.e. the decision-maker should carry goods 4, 5, 6, and 8 into the bag, and the best objective value is 110.4. Since the objective of the chance-constrained programming model is a function of parameter $\alpha$, the sensitivity of the optimal objective can be investigated with respect to different parameters. The following predetermined confidence levels are selected to test the sensitivity:

$$
\alpha=0.1,0.2,0.3,0.4,0.5,0.6,0.7,0.8,0.9,0.95 \text {. }
$$

The other predetermined confidence levels are set as follows:

$$
\beta=\gamma=0.9
$$

By choosing different confidence levels, $\alpha$, we obtain Table 2. From Table 2, we find out that $\alpha$ has an effect on the optimal solutions, so that the total weight of the UMKP increases when the confidence level decreases, which just coincides with Theorem 6 . For understanding this results better, Figure 3 is given. 
Table 2. List of $\alpha$-maximum pack when $\beta=\gamma=0.9$.

\begin{tabular}{cccccccc}
\hline $\boldsymbol{\alpha}$ & $\mathbf{1}-\boldsymbol{\alpha}$ & $\boldsymbol{\alpha}$-maximum pack & $\boldsymbol{V}^{*}$ & $\boldsymbol{\alpha}$ & $\mathbf{1}-\boldsymbol{\alpha}$ & $\boldsymbol{\alpha}$-maximum pack & $\boldsymbol{V}^{*}$ \\
\hline 0.95 & 0.05 & $(4,5,6,8)$ & 109 & 0.5 & 0.5 & $(4,5,6,8)$ & 116 \\
0.9 & 0.1 & $(4,5,6,8)$ & 110.4 & 0.4 & 0.6 & $(2,3,6,8)$ & 117.2 \\
0.8 & 0.2 & $(4,5,6,8)$ & 111.8 & 0.3 & 0.7 & $(2,3,6,8)$ & 118.4 \\
0.7 & 0.3 & $(4,5,6,8)$ & 113.2 & 0.2 & 0.8 & $(2,3,6,8)$ & 119.6 \\
0.6 & 0.4 & $(4,5,6,8)$ & 114.6 & 0.1 & 0.9 & $(2,3,6,8)$ & 120.8 \\
\hline
\end{tabular}

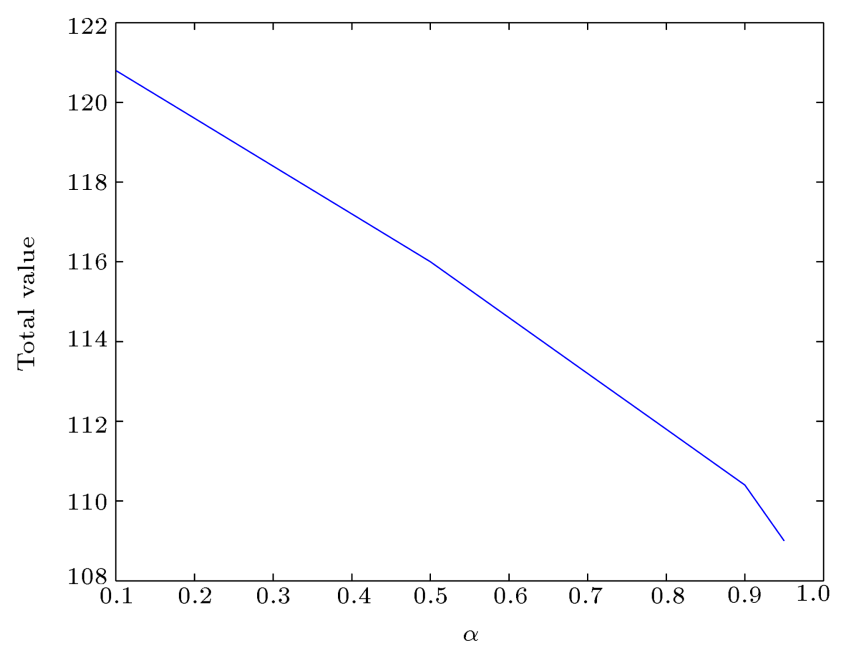

Figure 3. The sensitivity of the optimal value with different $\alpha$ of Example 3 .

Example 4. To further show the application of the models, more complex experiments have been done. Suppose that there are sixteen items and eight knapsacks. Let $m_{1}=0.7, m_{3}=0.8, m_{5}=0.85, m_{7}=0.9$, $m_{9}=0.9, m_{11}=0.95, m_{13}=0.8, m_{15}=0.9$, and $D=12$. In this numerical experiment, all uncertain parameters are assumed to be independent linear uncertain variables. The relevant uncertain parameters are shown in Tables 3-6.

Based on Theorem 4, the expected value model for UMKP is equivalent to the following model:

Table 3. Values, $\xi_{j}$, of sixteen items.

\begin{tabular}{cccccccc}
\hline $\boldsymbol{j}$ & $\boldsymbol{\xi}_{j}$ & $\boldsymbol{j}$ & $\boldsymbol{\xi}_{\boldsymbol{j}}$ & $\boldsymbol{j}$ & $\boldsymbol{\xi}_{\boldsymbol{j}}$ & $\boldsymbol{j}$ & $\boldsymbol{\xi}_{\boldsymbol{j}}$ \\
\hline 1 & $\mathcal{L}(32,36)$ & 5 & $\mathcal{L}(33,36)$ & 9 & $\mathcal{L}(34,40)$ & 13 & $\mathcal{L}(35,41)$ \\
2 & $\mathcal{L}(34,38)$ & 6 & $\mathcal{L}(34,36)$ & 10 & $\mathcal{L}(37,42)$ & 14 & $\mathcal{L}(38,42)$ \\
3 & $\mathcal{L}(32,39)$ & 7 & $\mathcal{L}(32,37)$ & 11 & $\mathcal{L}(34,35)$ & 15 & $\mathcal{L}(31,36)$ \\
4 & $\mathcal{L}(36,39)$ & 8 & $\mathcal{L}(36,38)$ & 12 & $\mathcal{L}(41,44)$ & 16 & $\mathcal{L}(42,46)$ \\
\hline
\end{tabular}

Table 4. Capacities $\zeta_{i}$ of eight knapsacks.

\begin{tabular}{ccccc}
\hline $\boldsymbol{i}$ & $\mathbf{1}$ & $\mathbf{2}$ & $\mathbf{3}$ & $\mathbf{4}$ \\
\hline$\zeta_{i}$ & $\mathcal{L}(42,102)$ & $\mathcal{L}(47,107)$ & $\mathcal{L}(50,120)$ & $\mathcal{L}(70,120)$ \\
\hline $\boldsymbol{i}$ & $\mathbf{5}$ & $\mathbf{6}$ & $\mathbf{7}$ & $\mathbf{8}$ \\
\hline$\zeta_{i}$ & $\mathcal{L}(50,100)$ & $\mathcal{L}(40,115)$ & $\mathcal{L}(40,100)$ & $\mathcal{L}(74,120)$ \\
\hline
\end{tabular}

Table 5. Discount coefficients $\tau_{j}$ of sixteen items.

\begin{tabular}{cccccccc}
\hline $\boldsymbol{j}$ & $\boldsymbol{\tau}_{\boldsymbol{j}}$ & $\boldsymbol{j}$ & $\boldsymbol{\tau}_{\boldsymbol{j}}$ & $\boldsymbol{j}$ & $\boldsymbol{\tau}_{\boldsymbol{j}}$ & $\boldsymbol{j}$ & $\boldsymbol{\tau}_{\boldsymbol{j}}$ \\
\hline 1 & $\mathcal{L}(3,5)$ & 5 & $\mathcal{L}(3,6)$ & 9 & $\mathcal{L}(5,10)$ & 13 & $\mathcal{L}(5,11)$ \\
2 & $\mathcal{L}(4,6)$ & 6 & $\mathcal{L}(3,6)$ & 10 & $\mathcal{L}(8,12)$ & 14 & $\mathcal{L}(8,13)$ \\
3 & $\mathcal{L}(2,7)$ & 7 & $\mathcal{L}(3,8)$ & 11 & $\mathcal{L}(3,12)$ & 15 & $\mathcal{L}(3,15)$ \\
4 & $\mathcal{L}(6,8)$ & 8 & $\mathcal{L}(6,11)$ & 12 & $\mathcal{L}(4,15)$ & 16 & $\mathcal{L}(4,14)$ \\
\hline
\end{tabular}

$$
\max \sum_{j=1}^{16} x_{j} \int_{0}^{1} \Phi_{j}^{-1}(\alpha) \mathrm{d} \alpha,
$$

subject to:

$$
\begin{aligned}
& \sum_{j=1}^{16} x_{j} \int_{0}^{1} \Psi_{i j}^{-1}(\alpha) \mathrm{d} \alpha \leq \int_{0}^{1} \Upsilon_{i}^{-1}(\alpha) \mathrm{d} \alpha, \\
& \quad \text { for } i=1,2, \cdots, 8, \\
& \sum_{j=1}^{16} y_{j} \int_{0}^{1} F_{j}^{-1}(\alpha) \mathrm{d} \alpha \geq D, \quad \text { for } j=1,2, \cdots, 16 .
\end{aligned}
$$

With the help of mathematical software LINGO, we can solve this $0-1$ programming problem (9). The result shows that the decision-maker should carry goods 2,3 , $5,7,9,12,13$, and 14 into the bag, and the expected maximum total value of the items that can be carried in the bag is 296 .

Assume that the confidence levels are $\alpha=\beta=$ $\gamma=0.9$. Based on Theorem 5, the chance-constrained programming model for UMKP is equivalent to the following one:

$$
\max V \text {, }
$$

subject to:

$$
\begin{aligned}
& \sum_{j=1}^{16} x_{j} \Phi_{j}^{-1}(0.1) \geq V \\
& \sum_{j=1}^{16} x_{j} \Psi_{i j}^{-1}(0.9) \leq \Upsilon_{i}^{-1}(0.1), \quad \text { for } i=1,2, \cdots, 8,
\end{aligned}
$$


Table 6. Weights, $\eta_{i j}$, of each item in each knapsack.

\begin{tabular}{ccccccccc}
\hline Items $/$ knapsack & $\mathbf{1}$ & $\mathbf{2}$ & $\mathbf{3}$ & $\mathbf{4}$ & $\mathbf{5}$ & $\mathbf{6}$ & $\mathbf{7}$ & $\mathbf{8}$ \\
\hline 1 & $\mathcal{L}(5,19)$ & $\mathcal{L}(6,11)$ & $\mathcal{L}(7,15)$ & $\mathcal{L}(5,13)$ & $\mathcal{L}(13,19)$ & $\mathcal{L}(8,11)$ & $\mathcal{L}(12,15)$ & $\mathcal{L}(9,13)$ \\
2 & $\mathcal{L}(5,13)$ & $\mathcal{L}(10,13)$ & $\mathcal{L}(5,12)$ & $\mathcal{L}(10,12)$ & $\mathcal{L}(9,13)$ & $\mathcal{L}(12,13)$ & $\mathcal{L}(8,12)$ & $\mathcal{L}(12,15)$ \\
3 & $\mathcal{L}(8,14)$ & $\mathcal{L}(8,17)$ & $\mathcal{L}(7,13)$ & $\mathcal{L}(6,13)$ & $\mathcal{L}(12,14)$ & $\mathcal{L}(13,17)$ & $\mathcal{L}(12,13)$ & $\mathcal{L}(6,13)$ \\
4 & $\mathcal{L}(5,13)$ & $\mathcal{L}(12,16)$ & $\mathcal{L}(10,17)$ & $\mathcal{L}(7,12)$ & $\mathcal{L}(9,14)$ & $\mathcal{L}(14,16)$ & $\mathcal{L}(13,17)$ & $\mathcal{L}(13,15)$ \\
5 & $\mathcal{L}(6,13)$ & $\mathcal{L}(7,15)$ & $\mathcal{L}(10,15)$ & $\mathcal{L}(5,7)$ & $\mathcal{L}(9,13)$ & $\mathcal{L}(12,15)$ & $\mathcal{L}(13,15)$ & $\mathcal{L}(7,10)$ \\
6 & $\mathcal{L}(10,17)$ & $\mathcal{L}(8,15)$ & $\mathcal{L}(10,14)$ & $\mathcal{L}(17,23)$ & $\mathcal{L}(12,17)$ & $\mathcal{L}(10,15)$ & $\mathcal{L}(11,14)$ & $\mathcal{L}(18,23)$ \\
7 & $\mathcal{L}(10,18)$ & $\mathcal{L}(5,13)$ & $\mathcal{L}(11,19)$ & $\mathcal{L}(11,15)$ & $\mathcal{L}(13,18)$ & $\mathcal{L}(10,13)$ & $\mathcal{L}(14,19)$ & $\mathcal{L}(15,19)$ \\
8 & $\mathcal{L}(10,16)$ & $\mathcal{L}(5,14)$ & $\mathcal{L}(17,19)$ & $\mathcal{L}(17,22)$ & $\mathcal{L}(12,16)$ & $\mathcal{L}(10,14)$ & $\mathcal{L}(18,19)$ & $\mathcal{L}(20,22)$ \\
9 & $\mathcal{L}(5,19)$ & $\mathcal{L}(6,11)$ & $\mathcal{L}(7,15)$ & $\mathcal{L}(5,9)$ & $\mathcal{L}(13,19)$ & $\mathcal{L}(8,11)$ & $\mathcal{L}(12,15)$ & $\mathcal{L}(9,13)$ \\
10 & $\mathcal{L}(5,13)$ & $\mathcal{L}(10,13)$ & $\mathcal{L}(5,12)$ & $\mathcal{L}(10,15)$ & $\mathcal{L}(9,13)$ & $\mathcal{L}(12,13)$ & $\mathcal{L}(8,12)$ & $\mathcal{L}(12,15)$ \\
11 & $\mathcal{L}(8,14)$ & $\mathcal{L}(8,17)$ & $\mathcal{L}(7,13)$ & $\mathcal{L}(6,13)$ & $\mathcal{L}(12,14)$ & $\mathcal{L}(13,17)$ & $\mathcal{L}(12,13)$ & $\mathcal{L}(9,14)$ \\
12 & $\mathcal{L}(5,13)$ & $\mathcal{L}(12,16)$ & $\mathcal{L}(10,17)$ & $\mathcal{L}(7,12)$ & $\mathcal{L}(9,13)$ & $\mathcal{L}(14,16)$ & $\mathcal{L}(13,17)$ & $\mathcal{L}(12,16)$ \\
13 & $\mathcal{L}(6,13)$ & $\mathcal{L}(7,15)$ & $\mathcal{L}(10,15)$ & $\mathcal{L}(5,10)$ & $\mathcal{L}(9,15)$ & $\mathcal{L}(12,15)$ & $\mathcal{L}(13,15)$ & $\mathcal{L}(7,10)$ \\
14 & $\mathcal{L}(10,17)$ & $\mathcal{L}(8,15)$ & $\mathcal{L}(10,14)$ & $\mathcal{L}(17,23)$ & $\mathcal{L}(12,17)$ & $\mathcal{L}(10,15)$ & $\mathcal{L}(11,14)$ & $\mathcal{L}(18,23)$ \\
15 & $\mathcal{L}(10,18)$ & $\mathcal{L}(5,13)$ & $\mathcal{L}(11,19)$ & $\mathcal{L}(11,15)$ & $\mathcal{L}(13,18)$ & $\mathcal{L}(10,13)$ & $\mathcal{L}(14,19)$ & $\mathcal{L}(15,19)$ \\
16 & $\mathcal{L}(10,16)$ & $\mathcal{L}(5,14)$ & $\mathcal{L}(17,19)$ & $\mathcal{L}(17,22)$ & $\mathcal{L}(12,16)$ & $\mathcal{L}(10,14)$ & $\mathcal{L}(18,19)$ & $\mathcal{L}(20,22)$ \\
\hline
\end{tabular}

$$
\sum_{j=1}^{16} y_{j} F_{j}^{-1}(0.1) \geq D
$$$$
x_{j} \in\{0,1\}, y_{j} \in\{0,1\}, \quad \text { for } j=1,2, \cdots, 16
$$

By using the mathematical software LINGO, the optimal solution to Model (10) can be obtained as $x_{1}=$ $x_{3}=x_{4}=x_{7}=x_{9}=x_{11}=x_{13}=x_{15}=1$, and the other remaining variables are equal to 0 . The optimal value of the objective is equal to 294.6.

If the weights, values, and capacities are general uncertain variables, then it is difficult for us to obtain their uncertainty distributions. Therefore, we can use uncertain statistics [21] to determine the uncertainty distribution. With the help of the designed questionnaire survey, the uncertainty distribution can be determined from the expert's experimental data by using the principle of least squares, the method of moments, the Delphi method, and so on [33].

\section{Conclusions and future research}

In the real-life industrial engineering applications, we usually encounter the uncertain factors due to lack of observed data about the unknown state of nature. Different from previous studies in indeterminacy environment, this paper mainly investigated multidimensional knapsack problem under uncertain environment with discount constraint, where the weights, values, and capacities of each item were depicted as uncertain variables. This work provided significant contributions to the existing research in the area of uncertain network optimization in which the relevant parameters might be characterized by uncertain variables and have significant implications in the real-world industrial engineering applications.

The main contributions of this paper include the following three aspects. Firstly, in order to deal with uncertain factors in multidimensional knapsack problem, the uncertainty theory was introduced into the problem under the light of uncertain environment. Secondly, two types of mathematical models for uncertain multidimensional knapsack problem were proposed according to two kinds of decision criteria, i.e. the expected value and the critical value criteria. We found out that the maximum value of chance-constrained programming model was actually decreasing with respect to the predetermined confidence level. Thirdly, some numerical examples were given to show the applications of the proposed models. In the numerical examples, we employed two kinds of uncertain variables, i.e. linear uncertain variables and zigzag uncertain variables, to characterize the weights, values, and capacities of the multidimensional knapsack problem. The numerical results illustrated that the proposed models are feasible and efficient for solving the multidimensional knapsack problem with uncertain parameters.

It should be noted that the proposed uncertain programming models in this paper have their unique advantages; that is, they can be easily transformed into their corresponding deterministic equivalent model which can be solved by the classical methods conveniently. In spite of these advantages, a few issues need to be addressed in the future. For example, we can continue to study the minimum weight dominating set problem and the maximum bi-section problem in 
uncertain environment within the axiomatic framework of uncertainty theory. In addition, the current work can be extended to the uncertain random environment $[34,35]$ where uncertainty and randomness coexist in a system. What's more, the situation in which the decision-makers adopt the minimax regret criterion $[32,36]$ to make the decisions in the uncertain multidimensional knapsack problem might be considered. While these issues beyond the scope of the present study, we believe they can be potential avenues for future studies.

\section{Acknowledgments}

This work was supported by the National Natural Science Foundation of China (No. 71671135), the Natural Science Foundation of Hubei Province (No. 2014CFC1096), the 2014 Key Project of Hubei Provincial Department of Education (No. D20142903), the Projects of the Humanity and Social Science Foundation of Ministry of Education of China (No. 13YJA630065), and the Key Project of Hubei Provincial Natural Science Foundation (No. 2015CFA144).

\section{References}

1. Rahmani, D., Yousefli, A. and Ramezanian, R. "A new robust fuzzy approach for aggregate production planning", Scientia Iranica, 21(6), pp. 2307-2314 (2014).

2. Tabrizi, B., Tavakkoli-Moghaddam, R. and Ghaderi, S. "A two-phase method for a multi-skilled project scheduling problem with discounted cash flows", Scientia Iranica, 21(3), pp. 1083-1095 (2014).

3. Partovi, F. and Seifbarghy, M. "Service centers location problem considering service diversity within queuing framework", Scientia Iranica, 22(3), pp. 11031116 (2015).

4. Ramezani, M., Kimiagari, A. and Karimi, B. "Interrelating physical and financial flows in a bi-objective closed-loop supply chain network problem with uncertainty", Scientia Iranica, 22(3), pp. 1278-1293 (2015).

5. Freville, A. "The multidimensional 0-1 knapsack problem: An overview", Eur. J. Oper. Res., 155(1), pp. 1-21 (2004).

6. Henig, M. "Risk criteria in a stochastic knapsack problem", Oper. Res., 38(5), pp. 820-825 (1990).

7. Kleywegt, A. and Papastavrou, J. "The dynamic and stochastic knapsack problem", Oper. Res., 46(1), pp. 17-35 (1998).

8. Kosuch, S. "Approximability of the two-stage stochastic knapsack problem with discretely distributed weights", Discret. Appl. Math., 165, pp. 192-204 (2014).

9. Feng, T. and Hartman, J. "The dynamic and stochastic knapsack problem with homogeneous-sized items and postponement options", Naval Res. Logist., 62(4), pp. 267-292 (2015).
10. Han, J., Lee, K., Lee, C., Choi, K. and Park, S. "Robust optimization approach for a chance-constrained binary knapsack problem", Math. Program., 157(1), pp. 277-296 (2016).

11. Gibson, M., Ohlmann, J. and Fry, M. "An agent-based stochastic ruler approach for a stochastic knapsack problem with sequential competition", Comput. Oper. Res., 37(3), pp. 598-609 (2010).

12. Merzifonluoglu, Y., Geunes, J. and Romeijn, H. "The static stochastic knapsack problem with normally distributed item sizes", Math. Program., 134(2), pp. 459489 (2012).

13. Okada, S. and Gen, M. "Fuzzy multiple choice knapsack problem", Fuzzy Sets Syst., 67(1), pp. 71-80 (1994).

14. Changdar, C., Mahapatra, G. and Pal, R. "An improved genetic algorithm based approach to solve constrained knapsack problem in fuzzy environment", Expert Syst. Appl., 42(4), pp. 2276-2286 (2015).

15. Changdar, C., Mahapatra, G. and Pal, R. "An ant colony optimization approach for binary knapsack problem under fuzziness", Appl. Math. Comput., 223, pp. 243-253 (2013).

16. Chen, S. "Analysis of maximum total return in the continuous knapsack problem with fuzzy object weights", Appl. Math. Modell., 33(7), pp. 2927-2933 (2009).

17. Baykasoğlu, K. and Ozsoydan, F. "An improved firefly algorithm for solving dynamic multidimensional knapsack problems", Expert Syst. Appl., 41(8), pp. 37123725 (2014).

18. Kasperski, A. and Kulej, K. "The 0-1 knapsack problem with fuzzy data", Fuzzy Optimization Decis. Mak., 6(2), pp. 163-172 (2007).

19. Kahneman, D. and Tversky, A. "Prospect theory: An analysis of decision under risk", Econometrica, 47(2), pp. 263-292 (1979).

20. Liu, B. "Why is there a need for uncertainty theory", J. Uncertain Syst., 6(1), pp. 3-10 (2012).

21. Liu, B., Uncertainty Theory: A Branch of Mathematics for Modeling Human Uncertainty, Springer, Verlag, Berlin (2010).

22. Liu, B., Uncertainty Theory, 2nd Edn., Springer, Verlag, Berlin (2007).

23. Peng, J. and Zhang, B. "Knapsack problem with uncertain weights and values", http://orsc.edu.cn/ online/120422.pdf (2012).

24. Zhang, B. and Peng, J. "Uncertain programming model for uncertain optimal assignment problem", Appl. Math. Modell., 37(9), pp. 6458-6468 (2013).

25. Gao, Y. "Shortest path problem with uncertain arc length", Comput. Math. Appl., 62(6), pp. 2591-2600 (2011).

26. Gao, Y., Yang, L. and Li, S. "Uncertain models on railway transportation planning problem", Appl. Math. Modell., 40(7-8), pp. 4921-4934 (2016). 
27. Liu, Z., Zhao, R., Liu, X. and Chen, L. "Contract designing for a supply chain with uncertain information based on confidence level", Appl. Soft Comput., 56, pp. 617-631 (2017). DOI: 10.1016/j.asoc.2016.05.054 (2016).

28. Liu, B., Theory and Practice of Uncertain Programming, 2nd Edn., Springer, Verlag, Berlin (2009).

29. Peng, J., Zhang, B. and Li, S. "Towards uncertain network optimization", J. Uncertainty Anal. Appl., 3(4), pp. 1-19 (2015).

30. Liu, B. "Some research problems in uncertainty theory", J. Uncertain Syst., 3(1), pp. 3-10 (2009).

31. Charnes, A. and Cooper, W. "Chance-constrained programming", Manage. Sci., 6(1), pp. 73-79 (1959).

32. Kasperski, A. and Zieliński, P. "Minmax regret approach and optimality evaluation in combinatorial optimization problems with interval and fuzzy weights", Eur. J. Oper. Res., 200(3), pp. 680-687 (2010).

33. Wang, X., Gao, Z. and Guo, H. "Uncertain hypothesis testing for two experts' empirical data", Math. Comput. Model., 55(3-4), pp. 1478-1482 (2012).

34. Liu, Y. "Uncertain random variables: A mixture of uncertainty and randomness", Soft Comput., 17(4), pp. 625-634 (2013).

35. Liu, Y. "Uncertain random programming with applications", Fuzzy Optimization Decis. Mak., 12(2), pp. 153-169 (2013).

36. Kasperski, A. and Zieliński, P. "A 2-approximation algorithm for interval data minmax regret sequencing problems with the total flow time criterion", Oper. Res. Lett., 36(3), pp. 343-344 (2008).

\section{Biographies}

Li Cheng is currently a Master Candidate of College of Mathematics and Physics at Huanggang Normal University, Hubei, China. Her research interests focus on uncertainty theory and its application to combinatorial optimization and higher education management.

Congjun Rao received his $\mathrm{PhD}$ degree in Huazhong University of Sciences and Technology, China. Now, he is an Associate Professor at Huanggang Normal University, Hubei, China. His general research topics of interest include: uncertainty theory, management decision-making, etc. He has published more than 50 papers in various journals, where 22 papers are indexed by SCI and SSCI.

Lin Chen received his MS degree in Applied Mathematics from Shanghai Normal University in 2015. Now, he is a PhD candidate in the College of Management and Economics, Tianjin University, China. His research interests include uncertain theory and its application to supply chain management and uncertain network optimization. His recent studies have been published in International Journal of Production Research, Applied Soft Computing, International Journal of Systems Science, and Journal of Intelligent Manufacturing, etc. 